\title{
$23 \%$ power conversion efficiency light-emitting diodes using monolayer quantum dots
}

Fengjia Fan ( $\nabla$ ffj@ustc.edu.cn )

University of Science and Technology of China

\section{Yan Gao}

Henan University

Xiaonan Liu

Henan University

\section{Bo Li}

University of Science and Technology of China

Huaibin Shen

Henan University

\section{Yang Song}

University of Science and Technology of China

Jiaojiao Song

Henan University

\section{Yihua Chong}

Henan University

Ruyun Yao

Henan University

\section{Shujie Wang}

Henan University

\section{Lin Song Li}

Henan University https://orcid.org/0000-0001-7015-3211

\section{Zuliang Du}

Henan University https://orcid.org/0000-0002-9780-7593

\section{Physical Sciences - Article}

Keywords: colloidal quantum dots, light-emitting diodes, monolayer quantum dots, efficiency

Posted Date: June 23rd, 2021

DOI: https://doi.org/10.21203/rs.3.rs-624492/v1 
License: (c) (i) This work is licensed under a Creative Commons Attribution 4.0 International License. Read Full License 


\section{$23 \%$ power conversion efficiency light-emitting diodes using monolayer quantum dots}

Yan Gao ${ }^{1}$, Xiaonan $\mathrm{Liu}^{1}$, Bo $\mathrm{Li}^{2}$, Huaibin Shen ${ }^{1 *}$, Yang Song ${ }^{2}$, Jiaojiao Song ${ }^{1}$, Yihua Chong ${ }^{1}$, Ruyun Yao ${ }^{1}$, Shujie Wang ${ }^{1}$, Lin Song $\mathrm{Li}^{1}$, Fengjia Fan ${ }^{2 *}$, and Zuliang Du ${ }^{1 *}$

$5{ }^{1}$ Key Laboratory for Special Functional Materials of Ministry of Education, National \& Local Joint Engineering Research Center for High-efficiency Display and Lighting Technology, Henan University, Kaifeng 475004, China.

${ }^{2}$ Hefei National Laboratory for Physical Sciences at the Microscale and Department of Modern Physics, CAS Key Laboratory of Microscale Magnetic Resonance, Synergetic Innovation Center of Quantum Information and Quantum Physics, University of Science and Technology of China, Hefei 230026, China

*e-mails: shenhuaibin@ henu.edu.cn; ffj@ustc.edu.cn; zld@ henu.edu.cn;

Ever since the first proposal of using colloidal quantum dots (QDs) as the active emitting layer of light-emitting diode (LED) ${ }^{1}$, a monolayer of QD is considered as a better option than the multilayer ones ${ }^{2,3}$. Owing to the slow charge transport rate among different QD layers, quantum dot light-emitting diodes (QLEDs) adopting multilayer QDs need to be driven at higher than the bandgap bias voltage to achieve practically useful brightness, resulting in increased power consumptions and heat generations, and reduced device lifetimes ${ }^{4-11}$. Unfortunately, QLEDs using monolayer QDs always suffer from unwanted recombination in hole transport layers (HTLs) and low external quantum efficiencies (EQEs) as a result of electron overflow from QDs into HTLs ${ }^{2,3,12}$. Herein, we tackle this dilemma by packing QDs with large size into monolayers, which enables us to mitigate the unwanted electron overflow and retain high EQE. More importantly, it further allows us to boost the irradiative recombination current at bandgap voltage. By virtue of simultaneously obtained high EQE and irradiative recombination rate, we can achieve brightness of $1,100 \mathrm{~cd} \mathrm{~m}^{-2}$ and $3,000 \mathrm{~cd} \mathrm{~m}^{-}$ 
${ }^{2}$ at $100 \%$ and $105 \%$ bandgap voltages with record high power conversion efficiencies (PCEs) of $23 \%$ and $22 \%$, respectively. Since heat generation has been depressed and devices can be operated at reduced bias voltage, they show unprecedented $T_{95}$ operation lifetimes (the time for the luminance to decrease to $95 \%$ of the initial value) of more than $4,000 \mathrm{~h}$ with an initial brightness of 3,000 $\mathrm{cd} \mathrm{m}^{-2}$, and equivalent $T_{95}$ lifetimes of more than $20,000 \mathrm{~h}$ at $1,000 \mathrm{~cd} \mathrm{~m}^{-2}$.

It is the PCE that determines how energy efficient the QLEDs are, and high PCEs simultaneously require high EQEs and large irradiative recombination currents at low bias voltages ${ }^{13}$. Current state of art QLEDs ubiquitously adopt multiple QD layers, they are featured with high peak EQEs but small recombination currents around the bandgap voltages ${ }^{4-12}$. To achieve a brightness ranging from 1,000 to $3,000 \mathrm{~cd} \mathrm{~m}^{-2}$ - one that required for flat panel and outdoor displays $^{14,15}$, bias voltages that noticeably higher than the bandgap voltage need to be applied. Despite near unity internal quantum efficiencies, the high bias voltages limit the power conversion efficiency (PCE) to 5-18\% $\%^{4-11}$ - values well below the EQE.

The small irradiative recombination currents in multilayer QDs are mainly due to the slow charge carrier transport rate among different QD layers ${ }^{16,17}$. In theory, this issue can be circumvented in monolayer QDs because charge carriers are injected directly from the charge transport layers without the necessity to transport among different layers. However, early attempts to use monolayer QDs as active emitting layers turn out to be unsuccessful, the electron overflow into HTLs leads to unwanted recombination which significantly impacts the $\mathrm{EQE}^{2,3,12}$.

To further investigate the electron overflow issue and seek remedies, we fabricated QLEDs using monolayers of regular CdSe-CdZnSe-ZnS QD with a mean size of $10 \mathrm{~nm}^{5,6,9}$ (Figure 1a, b and Extended Data Fig. 1). These devices show noticeable current leakage before they are turned on. Since hole injection is less efficient below the turn on voltage, that leakage current is mostly contributed from electron (Extended Data Fig. 2). With a 3 V bias voltage, weak electroluminance (EL) from HTL is observed (Figure 1c). Therefore, we conclude that electron overflow or leakage is present both below and above the turn on voltage, and moderate EQEs up to $14.5 \%$ are obtained despite the over 90\% photoluminescence quantum yield (PLQY) of QD. When we increase the thickness of QD layers, electron leakage can be mitigated and EQE can be improved to $20 \%$ (Extended Data Fig. 3); however, this is accompanied by more than 4 times decrement in recombination current. 
We therefore concluded that adopting a monolayer of $\mathrm{QD}$ is critical to achieve high recombination current, but depressing the electron overflow is a must to get high $\mathrm{EQE}$. There is a positive correlation between the electron overflow probability and electron concentration ${ }^{18}$, hence, it is reasonable to postulate that reducing the charge concentration in the QD monolayer will be beneficial in depressing the electron overflow. The total charge carrier concentration in the QD layer is determined by the number of QD per volume and charge population in each individual QDs. Since the latter is determined by quasi-Fermi level which is controlled by bias voltage ${ }^{19}$, the total charge carrier concentration at a given bias voltage can be decreased by reducing the QD number. Previous researches have demonstrated that by using a blend of $\mathrm{QDs}^{20}$ or QD in matrix ${ }^{21,22}$, the number of QD per volume can be reduced, leading to decreased turn-on voltages and improved quantum efficiencies. Herein, we opt to increase the QD size while still adopting monolayer packing, the exciton generation rate can be maximized by doing so because charge carriers are injected directly from charge transport layers into QDs without the necessity to transport among QDs.

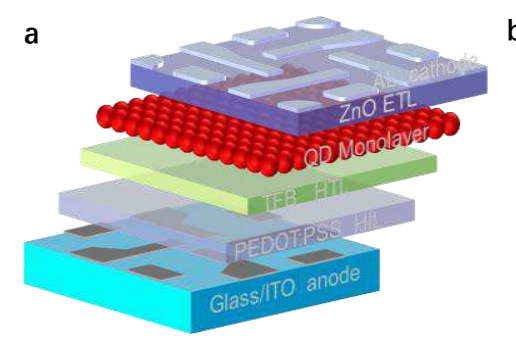

b
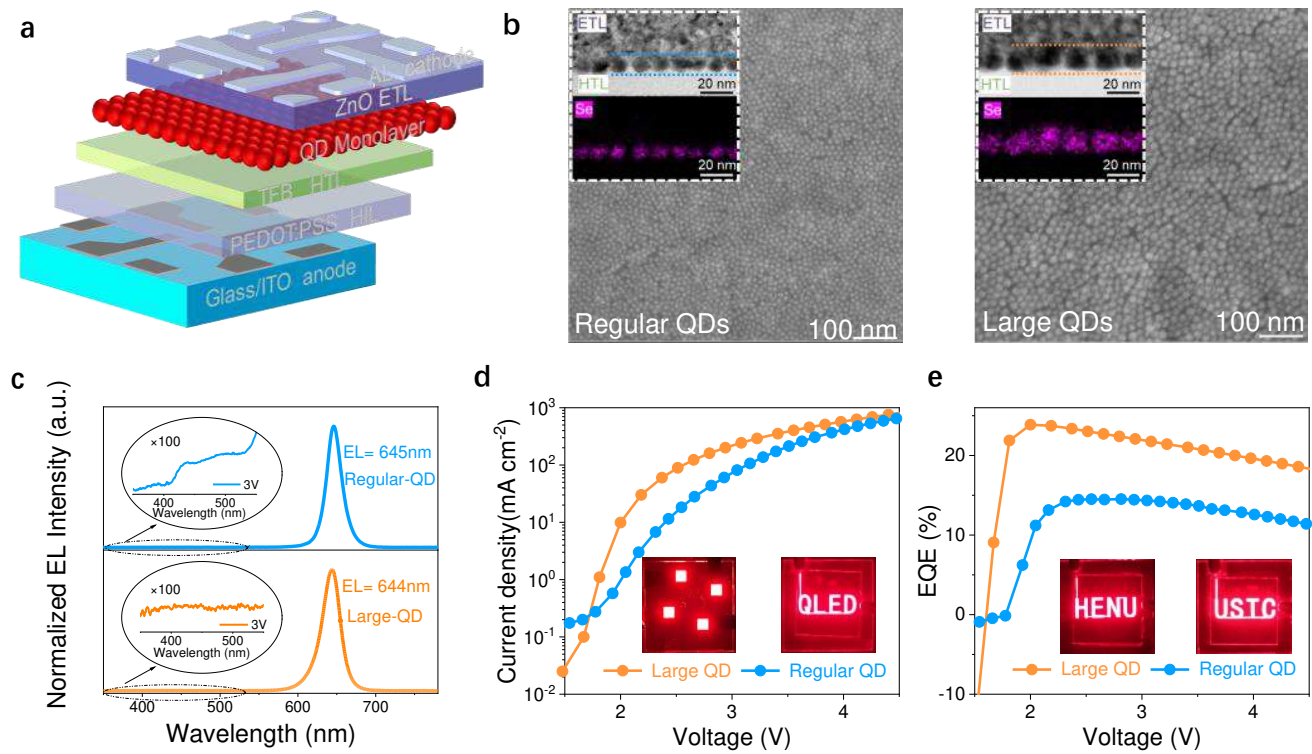

d

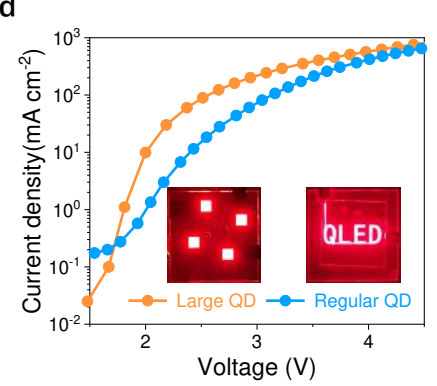

e

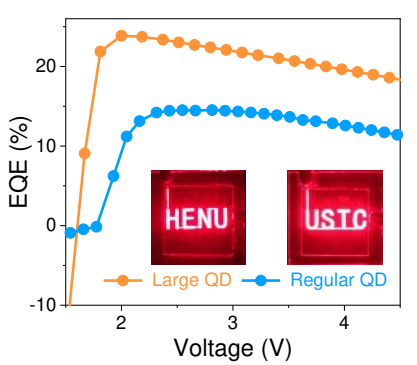

Figure 1| Characterizations of QLEDs using monolayer regular and large QDs. a, scheme of

QLED structure. b, sectional transmission electron microscopy (TEM) images of QLEDs, and scanning electron microscopy (SEM) images of QD monolayers on HTL. c, Similar emission peak positions are observed in EL spectra of QLEDs using monolayer QDs with average sizes of 10 and $18 \mathrm{~nm}$. Insets show 100 times zoomed in views of EL spectra from HTL. In QLED using regular QDs (10 nm in size), noticeable EL from HTL is observed, but it is efficiently eliminated when large QDs (18 nm in size) are used instead. d, the current-voltage curves of QLEDs. Below the turn-on 
voltage, the current leakage is efficiently depressed in QLED using large QDs, but conversely, irradiative recombination current above turn-on voltage is remarkably enhanced. e, the EQE of QLED using large QD is on par with that of the state-of-the-art QLEDs employing multiple QD layers, while the EQE in QLED using monolayer regular QD is much lower.

We then sought to synthesize QD with big size while maintaining high quantum yield. In previous literatures, the quantum yield of giant-shell QD are generally low ${ }^{23}$. It is either caused by delocalized electron wavefunction due to the quasi type-II band alignment or abundant trap states induced by the accumulated compressive $\operatorname{strain}^{23}$. To circumvent these two issues, we developed a one-pot protocol to synthesize continuously gradient type-I core-shell CdSe-ZnCdSe-ZnS QD with an average size of $18 \mathrm{~nm}$ (Extended Data Fig. 4). Thanks to the continuously alloyed shell, the interface strain is greatly mitigated, enabling us to achieve $>90 \%$ photoluminescence quantum yield. Uniform size distribution further allows narrow spectral linewidth (Extended Data Fig. 4).

We then fabricated QLEDs using monolayers of newly developed large QDs (Figure 1c, Extended Data Fig. 5), we found that both EL from HTL (Figure 1c) and the current leakage (Figure 1d) can be efficiently depressed, and EQE is boosted to $23.8 \%$ (Figure 1e), close to the out-coupling efficiency limit we calculated $(\sim 24.4 \%)^{24}$ (see Method, outcoupling efficiency simulation, Extended Data Table 1 and Extended Data Fig. 6). Even more strikingly, compared with QLED using monolayer regular QD, one that employs monolayer of large QD shows up to 8 times higher recombination current when the bias voltages are slightly above the turn-on voltage. But we indeed noticed that the difference diminishes when the driving voltage is above $4.5 \mathrm{~V}$ (Figure 1d).

Previous studies have found that hole injection barrier played an important role in determining the recombination current and $\mathrm{EQE}^{5,6}$. To investigate whether our QLEDs using large QDs are benefited from improved hole injections, we performed the ultraviolet photoemission spectroscopy (UPS) and single carrier conductance measurements (Extented Data Fig. 7, Extented Data Fig. 2). Both the valence band levels and hole injection currents in QLEDs using two different QDs are quite close to each other, therefore, we conclude that increased recombination currents are stemmed from different recombination dynamics rather than reduced hole injection barrier. 

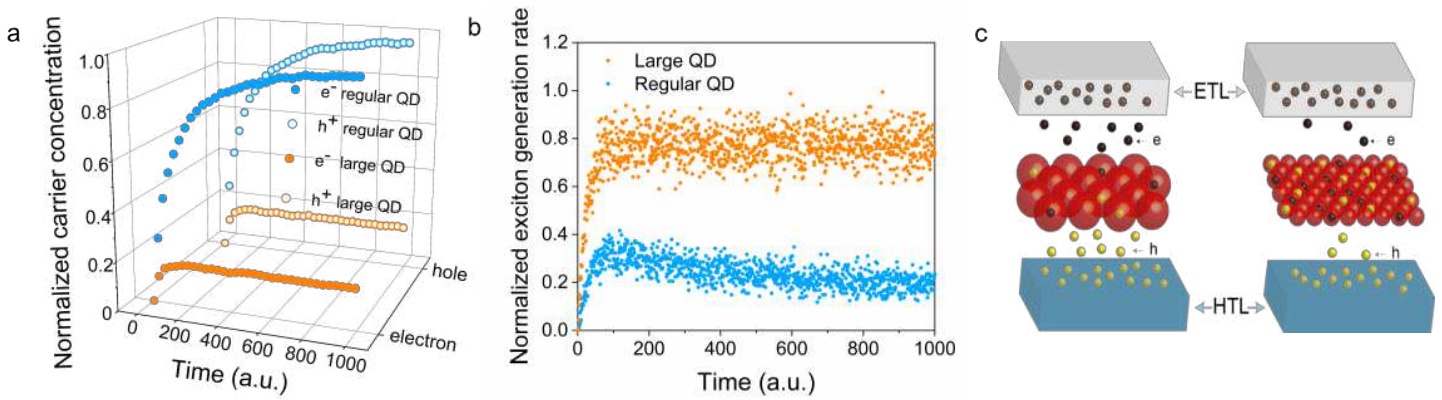

Figure 2| The Monte Carlo simulations. Time evolutions of normalized charge concentrations (a) and exciton generation rates (b) in QLEDs using monolayers of large and regular QDs, respectively. c, the scheme elucidates the origin of difference in recombination current. The charge carrier injection in QLED is dominated by the diffusion current around turn-on voltage, because the drift current is negligible due to the flat-band condition ${ }^{19}$. A lower charge carrier concentration in QD layer leads to a bigger charge carrier concentration gradient, and hence bigger charge injection current.

In quantum confined and poorly conductive QD films, the electrons and holes are injected into individual QDs, and they either stay inside the single QD or transport to the nearby QDs at slow rate, and thus they don't follow the traditional second order kinetics as bulk semiconductors do ${ }^{19}$. We therefore build a Monte Carlo model to simulate the carrier injection, diffusion and recombination dynamics (see Methods for the detailed information) in QLED. The emitting layer are separated into small areas to represent the individual QDs, and the vertical charge injection rate is dependent on the concentration gradient between the QD layers and charge transport layers. The excitons are mostly generated through direct vertical charge injections from charge transport layers to the oppositely charged QDs, and there is also some contribution from lateral charge migration among QDs (Extended Data Fig. 8). The vertical electrical currents in the active emitting layers are generated by irradiative recombination, while at their interface with charge transport layers, currents are contributed by charge diffusion. According to the continuity equation of electrical currents, they are equivalent to each other at steady sate ${ }^{19}$.

There are two important findings from this model. The first one is that at steady state, with given charge concentrations in transport layers, the total charge concentrations in large QD are indeed smaller than those in the regular ones, this aligns well with our hypothesis and explains the better depression of electron overflow in large QDs (Figure 2a). The second one is that the QLED 
using large QD shows much higher recombination current when the charge carrier concentrations in transport layers are relatively low, which agrees with what we experimentally observed with low bias voltages. We attribute the increased injection currents to the bigger concentration gradients between the transport and the QD layers. We also noticed that when the charge carrier concentrations in charge transport layers are high, the diffusion currents in two types of device resemble each other (Extended Data Fig.9, 10). This is because when charge concentrations in transport layers are overwhelmingly larger than that in the QD layers, the concentration gradient is largely determined by the carrier concentration in the charge transport layers. This result agrees well with our observation that when the applying voltage is higher than $4.5 \mathrm{~V}$, the difference in recombination current diminishes in two types of QLED.

a
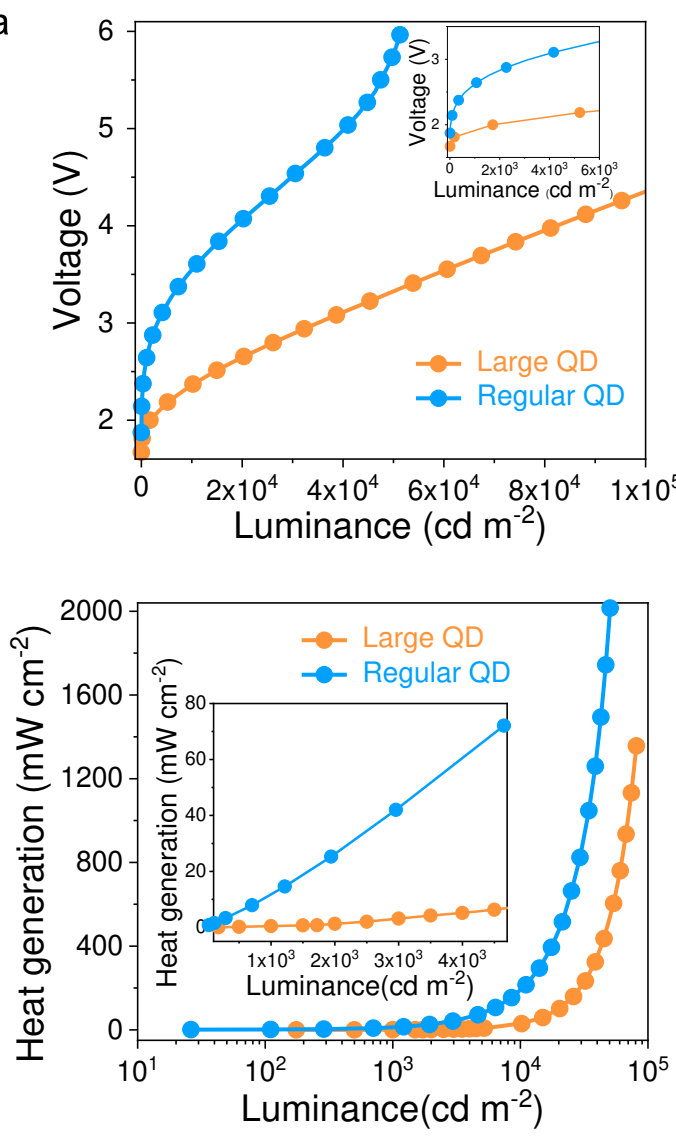
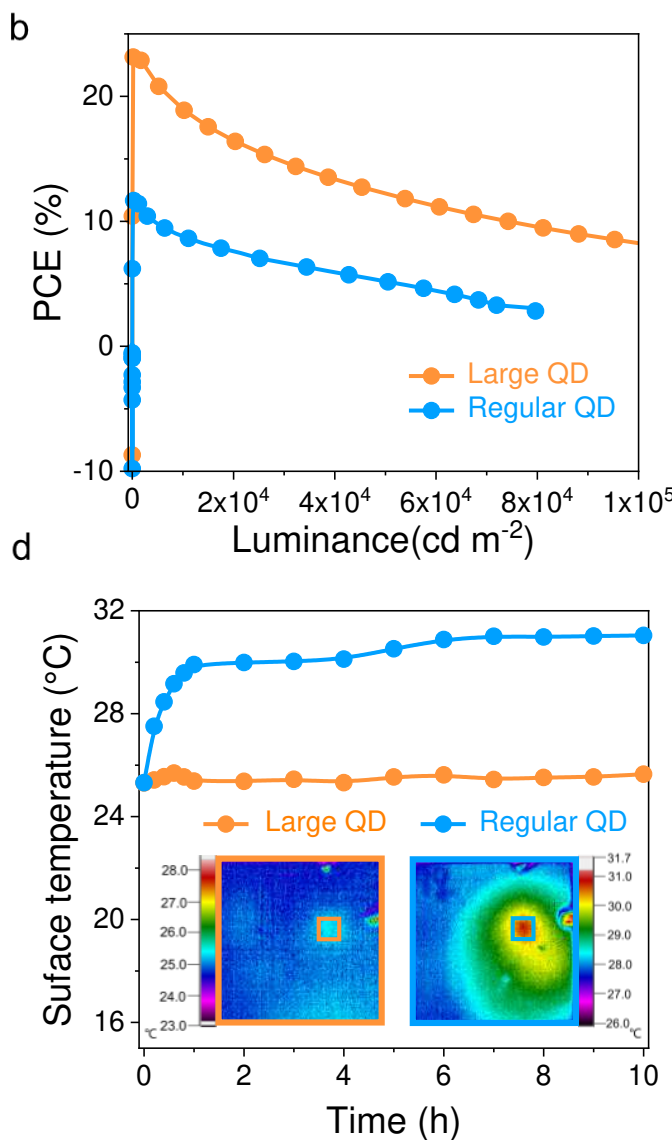

Figure 3| Power conversion efficiencies and heat generation rates. The dependence of bias voltage (a), power conversion efficiency (b), and heat generation rate (c) on luminance of QLEDs using monolayer large and regular QDs, respectively. $\mathbf{d}$, time evolution of surface temperature of QLEDs. 
As discussed above, high $\mathrm{EQE}$ and large irradiative recombination current have been simultaneously achieved in our QLED using monolayer large QDs, which enables us to achieve brightness of 1,100 and $3000 \mathrm{~cd} \mathrm{~m}^{-2}$ at the $100 \%$ and $105 \%$ bandgap voltages (Figure 3a), respectively. In contrast, our QLEDs using monolayer regular QD show much lower brightness at both the $100 \%$ and $105 \%$ bandgap voltages, which are 10 and $27 \mathrm{~cd} \mathrm{~m}^{-2}$ (Figure 3a), respectively. In previously reported high performance QLEDs, brightness of $10-600 \mathrm{~cd} \mathrm{~m}^{-2}$ can be obtained at the bandgap voltages (Extended Data Table 2) ) $^{4-11}$, and to achieve a brightness of $3000 \mathrm{~cd} \mathrm{~m}^{-2}$ these devices need to be operated at more than $125 \%$ bandgap voltage. Due to the decreased driving voltage, the power conversion efficiencies of QLEDs using large QDs have also been remarkably improved. They can retain high power conversion efficiencies of 22-23\% (Figure 3b) at brightness ranging from $1,000-3,000 \mathrm{~cd} \mathrm{~m}^{-2}$, in contrast to previously reported highest values of $17-18 \% 25$ (Extended Data Table 3), and 10-11\% and 14-16\% obtained in QLED using monolayer and three layers of regular QDs (Extended Data Fig.11), respectively.

In LEDs, the input electrical energy is either converted into photons or heat, the latter can be estimated by calculating the theoretical photon outcoupling efficiency, which are $22.4 \%$ and $24.4 \%$ for our QLEDs using regular and large QDs, respectively (see Methods for detailed transfer matrix method calculations, Extended Data Figure 6). Our QLEDs using regular and large QD are estimated to generate $40 \mathrm{mWcm}^{-2}$ and $3 \mathrm{mWcm}^{-2}$ heat flow at a brightness of $3000 \mathrm{cdm}^{-2}$ (Figure 3c), respectively. Provided that ETL, HTL, QD film, and glass substrates are all poorly thermally conductive materials, a larger heat flow will inevitably heat QLED devices to a higher temperature ${ }^{26,27}$. We have continuously monitored the surface temperature of two types of QLED operating with a brightness of 3,000 $\mathrm{cd} \mathrm{m}^{-2}$ (Figure 3d). By virtue of the high power conversion efficiency in QLED using large QDs, there is negligible temperature rise after 10 hours operation at room temperature $\left(25^{\circ} \mathrm{C}\right)$; however, in QLED using regular QD, the surface temperature reaches $30{ }^{\circ} \mathrm{C}$ within half hours and stabilizes at $30-311^{\circ} \mathrm{C}$ after 10 hours operation. 

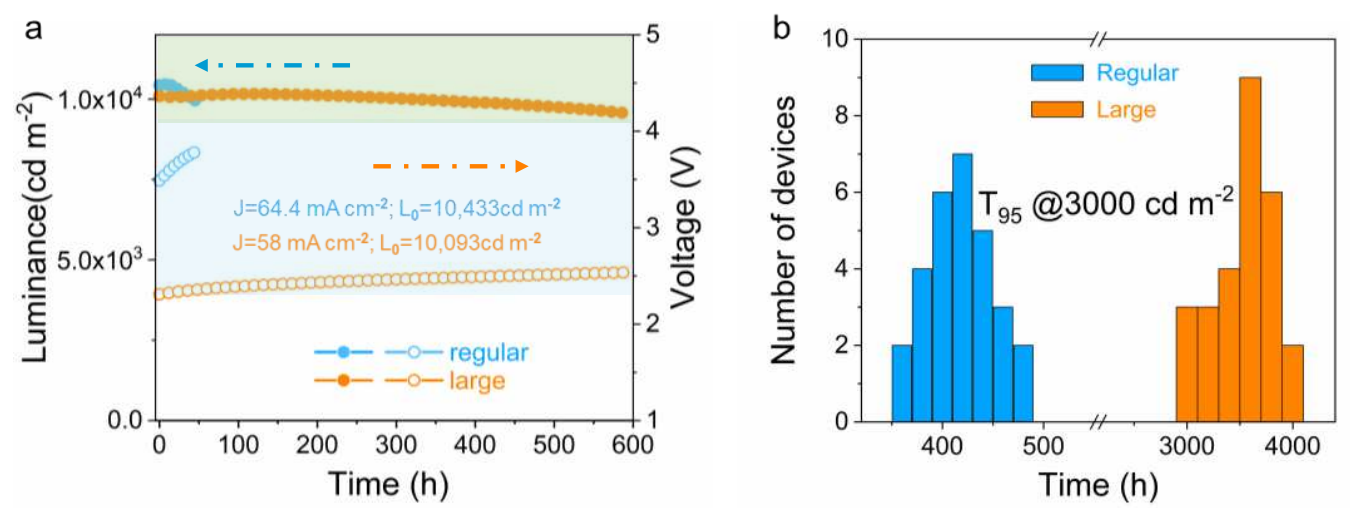

Figure $4 \mid$ Lifetime tests. a, $\mathrm{T}_{95}$ operational lifetime tests of QLED using large and regular QDs. The devices were tested under ambient conditions (temperature, $20-25^{\circ} \mathrm{C}$; relative humidity, 30 $50 \%)$. b, Histogram of estimated $\mathrm{T}_{95}$ lifetimes with an initial luminance of $3,000 \mathrm{~cd} \mathrm{~m}^{-2}$ from 27 and 29 QLEDs using large and regular QDs, respectively.

Regarding the stability of the QLEDs, electrochemical instabilities of ligand and HTL have been identified as the origins for device degradation ${ }^{8,28,29}$. From chemical dynamics point of view, all chemical reactions that account for the device degradation will be accelerated at alleviated temperatures. That is so say, regardless of the exact chemical mechanism, reducing heat generation in QLED can, in theory, mitigate the aging process of QLED. Recently, it has been reported that the lifetimes of QLEDs can be remarkably improved by using sapphire as substrate ${ }^{29}$ to dissipate heat better; however, this is not a cost-effective approach, and furthermore, heat accumulation can't be completely avoided because there are still poorly thermally conductive HTL and ETL. Herein, we propose to improve the power conversion efficiency as a strategy to tackle heating induced accelerated aging at the source.

We then turned to verify whether improved power conversion efficiency could enable better device stability. Our testing results reveal that the QLED using regular QD show a $\mathrm{T}_{95}$ lifetime of about $48 \mathrm{~h}$ at 10,433 $\mathrm{cd} \mathrm{m}^{-2}$ (Figure 4a). Fitting the lifetimes at different brightness with an empirical equation $\left(\mathrm{L}_{0}\right)^{\mathrm{n}} \times \mathrm{T}_{95}=$ constant $^{30}\left(\mathrm{~L}_{0}\right.$ is the initial brightness $)$, we obtained an acceleration factor of $\mathrm{n} \approx 1.74$ (Extended Data Fig. 12a). The $\mathrm{T}_{95}$ operational lifetimes at an initial brightness of 3,000 and $1,000 \mathrm{~cd} \mathrm{~m}^{-2}$ are then estimated to be around 400 and $2,500 \mathrm{~h}$, respectively, which are already comparable to those high performance QLEDs reported so far (Extended Data Table 4). Thanks to the unprecedented power conversion efficiency, QLEDs using large QD show impressive $\mathrm{T}_{95}$ lifetimes of $590 \mathrm{~h}$ operated at $10,093 \mathrm{~cd} \mathrm{~m}^{-2}$ (Figure 4a). With an acceleration factor of $\mathrm{n} \approx 1.58$ 
(Extended Data Fig. 12b), the $\mathrm{T}_{95}$ operational lifetimes at an initial brightness of 1,000 and 3,000 cd $\mathrm{m}^{-2}$ are estimated to be more than 22,000 and 4,000 h, respectively, which are about one order of magnitude longer than that of QLEDs using regular QD and more than 2 times longer than that of

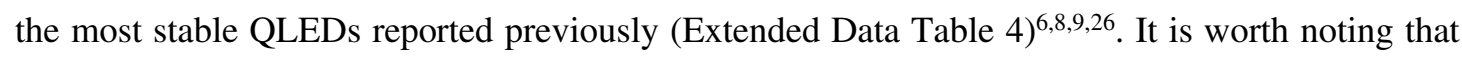
the obtained long lifetime here has already surpassed the industrial requirement for high-brightness displays applications ${ }^{9}$. Histograms of the lifetimes from more than 25 QLEDs in Figure 4c exhibit $\mathrm{T}_{95}$ lifetimes ranging from 3,000 to $4,000 \mathrm{~h}$ with a mean of $\sim 3,300 \mathrm{~h}$, suggesting high device fabrication reproducibility.

In conclusion, we have proposed to use monolayers of large size QDs to simultaneously increase the injection current and retain high EQE in QLEDs. This strategy enables us to achieve unprecedented high power conversion efficiencies of $22-23 \%$ at brightness ranging from 1000 to $3000 \mathrm{~cd} \mathrm{~cm}^{-2}$. This strategy also allows us to the mitigated heat generation and improve device operational lifetime. Long $\mathrm{T}_{95}$ lifetimes of 22,000 $\mathrm{h}$ and 4,000 $\mathrm{h}$ at luminance of 1,000 and 3,000 cd $\mathrm{m}^{-2}$ have been achieved respectively.

\section{Reference}

1. Colvin, V. L., Schlamp, M. C. \& Alivisatos, A. P. Light-emitting diodes made from cadmium selenide nanocrystals and a semiconducting polymer. Nature 370, 354-354 (1994).

2. Coe, S., Woo, W. K., Bawendi, M. \& Bulović, V. Electroluminescence from single monolayers of nanocrystals in molecular organic devices. Nature 420, 800-803 (2002).

3. Zhao, J. et al. Efficient $\mathrm{CdSe} / \mathrm{CdS}$ quantum dot light-emitting diodes using a thermally polymerized hole transport layer. Nano Lett. 6, 463-467 (2006).

4. Dai, X. et al. Solution-processed, high-performance light-emitting diodes based on quantum dots. Nature. 515, 96-99 (2014)

25 5. Yang, Y. et al. High-efficiency light-emitting devices based on quantum dots with tailored nanostructures. Nat. Photon. 9, 259-266 (2015).

6. Shen, H. et al. Visible quantum dot light-emitting diodes with simultaneous high brightness and efficiency. Nat. Photon. 13, 192-197 (2019).

7. Mashford, B. S. et al. High-efficiency quantum-dot light-emitting devices with enhanced charge 30 injection. Nat. Photon. 7, 407-412 (2013).

8. $\mathrm{Pu}, \mathrm{C}$. et al. Electrochemically-stable ligands bridge the photoluminescence-electroluminescence gap of quantum dots. Nat. Commun. 11, 937 (2020).

9. Cao, W. et al. Highly stable QLEDs with improved hole injection via quantum dot structure tailoring. Nat. Commun. 9, 2608 (2018).

35 10. Lin, J. et al. High-performance quantum-dot light-emitting diodes using $\mathrm{NiO}_{\mathrm{x}}$ hole-injection layers 
with a high and stable work function. Adv. Funct. Mater. 30, 1907265 (2020).

11. Li, X. et al. Bright colloidal quantum dot light-emitting diodes enabled by efficient chlorination. Nat. Photon. 12, 159-164 (2018).

12. Sun, Q.et al. Bright, multicoloured light-emitting diodes based on quantum dots. Nat. Photon. 1, 717-722 (2007).

13. Dai, X., Deng, Y., Peng, X. \& Jin, Y. Quantum-dot light-emitting diodes for large-area displays: towards the dawn of commercialization. Adv. Mater. 29, 1607022 (2017).

14. Shirasaki, Y., Supran, G. J., Bawendi, M. G. \& Bulović, V. Emergence of colloidal quantum-dot light-emitting technologies. Nat. Photon. 7, 13-23 (2013).

15. Quan, L. N., De Arquer, F. P. G., Sabatini, R. P. \& Sargent, E. H. Perovskites for Light Emission. Adv. Mater. 30, 1801996 (2018).

16. Shen, H. et al. High-efficiency, low turn-on voltage blue-violet quantum-dot-based light-emitting diodes. Nano Lett. 15, 1211-1216 (2015).

17. Sun, L. et al. Bright infrared quantum-dot light-emitting diodes through inter-dot spacing control. Nat. Nanotech. 7, 369-373 (2012).

18. Arkhipov, V. I., Emelianova, E. V., Tak, Y. H. \& Bssler, H. Charge injection into light-emitting diodes: Theory and experiment. J. Appl. Phys. 84, 848-856 (1998).

19. Neamen, D. A. Semiconductor Physics and Devices: Basic Principles, New York, McGraw-Hill, (2012).

20. Pradhan, S. et al. High-efficiency colloidal quantum dot infrared light-emitting diodes via engineering at the supra-nanocrystalline level. Nat. Nanotech.. 14, 72-79 (2019).

21. Gong, X. et al. Highly efficient quantum dot near-infrared light-emitting diodes. Nat. Photon. 10, 253-257 (2016).

22. Gao, L. et al. Efficient near-infrared light-emitting diodes based on quantum dots in layered perovskite. Nat. Photon. 14, 227-233 (2020).

23. Acharya, K. P. et al. Elucidation of two giants: challenges to thick-shell synthesis in CdSe/ZnSe and ZnSe/CdS core/shell quantum dots. J. Am. Chem. Soc. 137, 3755-3758 (2015).

24. Neyts, K. A. Simulation of light emission from thin-film microcavities. J. Opt. Soc. Am. A 15, $962-$ 971 (1998).

30 25. Liu, D. et al. Highly stable red quantum dot light-emitting diodes with long $\mathrm{T}_{95}$ operation lifetimes. J. Phys. Chem. Lett. 11, 3111-3115 (2020).

26. Ong, W.-L., Rupich, S. M., Talapin, D. V., McGaughey, A. J. H. \& Malen, J. A. Surface chemistry mediates thermal transport in three-dimensional nanocrystal arrays. Nat. Mater. 12, 410-415 (2013).

27. Liu, J. et al. Thermal Conductivity and Elastic Constants of PEDOT:PSS with High Electrical 35 Conductivity. Macromolecules 48, 585-591 (2015).

28. Chen, S. et al. On the degradation mechanisms of quantum-dot light-emitting diodes. Nat. Commun. 10,765 (2019).

29. Sun, Y., Su, Q., Zhang, H., Wang, F. \& Chen, S. Investigation on thermally induced efficiency rolloff: towards efficient and ultra-bright quantum-dot light-emitting diodes. ACS Nano 13, 11433-11442 (2019).

30. Scholz, S., Kondakov, D., Lüssem, B. \& Leo, K. Degradation mechanisms and reactions in organic 
light-emitting devices. Chem. Rev. 115, 8449-8503 (2015).

\section{Acknowledgements}

We gratefully acknowledge the financial support from the National Natural Science Foundation of

5 China (grant numbers 61922028, 81788101, 11761131011, 51872275, 61874039), the National Key R\&D Program of China (Grant No. 2018YFA0306600), the CAS (Grants No. GJJSTD20170001 and No. QYZDY-SSW-SLH004), the Anhui Initiative in Quantum Information Technologies (Grant No. AHY050000). and the Innovation Research Team of Science and Technology in Henan Province (20IRTSTHN020).

\section{Author contributions}

Z.L.D. took lead of the project. H.B.S and F.J.F. conceived the concept and designed the experiments. H.B.S., F.J.F and Z.L.D. supervised the project. Y.G, X.N.L and B.L contributed equally. Y.G, X.N.L and J.J.S synthesized the materials, fabricated the devices and collected the performance data of the QLEDs. B.L and Y.S developed the Monte Carlo model. F.J.F and H.B.S. wrote the manuscript. All authors contributed to the scientific discussion and modifying the manuscript.

\section{Competing interests}

The authors declare no competing interests

\section{Additional information}

Supplementary information is available for this paper at $\mathrm{xxxxxx}$.

Reprints and permissions information is available at www.nature.com/reprints. Correspondence and requests for materials should be addressed to H.B.S., F.J.F and Z.L.D. Publisher's note: Springer Nature remains neutral with regard to jurisdictional claims in published maps and institutional affiliations. 


\section{Methods}

Materials. Cadmium oxide (CdO, 99.99\%), zinc oxide (ZnO, 99.9\% powder), sulfur (S, 99.998\%, powder), 1-octadecene (ODE, 90\%), oleic acid (OA, 90\%), trioctylphosphine (TOP,97\%), selenium (Se, 99.99\%, powder), 1-octanethiol (OT, 98\%), chlorobenzene (99\%), zinc acetate dehydrate (98\%) as well as various solvents were purchased from Aldrich and Adamas-beta. dimethyl sulphoxide (DMSO, HPLC grade) were purchased from J\&K Chemical Ltd. poly(9,9-dioctylfluorene-co-N-(4(3-methylpropyl))-diphenylamine) (TFB) was purchased from American Dye Source. poly (ethylenedioxythiophene):polystyrene sulphonate poly (ethylenedioxythiophene): polystyrene sulphonate was purchased from Heraeus Deutschland GmbH \& Co.KG. All materials were used as received.

Preparation of the precursors: $\mathrm{Zinc}$ oleate $\left(\mathrm{Zn}(\mathrm{OA})_{2}\right)$ precursor: a mixture of $\mathrm{ZnO} 60 \mathrm{mmol}$, OA $(60 \mathrm{~mL})$, and ODE $(90 \mathrm{~mL})$ was loaded into a $250 \mathrm{~mL}$ three-neck flask. The mixture was then degassed at $150{ }^{\circ} \mathrm{C}$ for $15 \mathrm{~min}$. Finally, the solution was heated to $310{ }^{\circ} \mathrm{C}$ under nitrogen with vigorous stirring to get a colorless clear solution. Solution for Se precursor I: it was made by degassing Se (1.578 g, $20 \mathrm{mmol}), 40 \mathrm{~mL}$ of TOP in a $100 \mathrm{~mL}$ three-necked flask, then it was heated to $150{ }^{\circ} \mathrm{C}$ until the solution turn into transparent. Solution of Se precursor II: it was made by degassing Se (1.578 g, $20 \mathrm{mmol}), 100 \mathrm{~mL}$ of ODE in a $250 \mathrm{~mL}$ three necked flask, then it was heated to $220{ }^{\circ} \mathrm{C}$ until the solution turn into transparent light yellow ${ }^{31}$.

Synthesis of CdSe/CdZnSe/ZnS QDs: CdSe/CdZnSe/ZnS core/shell QDs were prepared by mixing $1.6 \mathrm{mmol} \mathrm{CdO}, 4 \mathrm{mmol} \mathrm{Zn}(\mathrm{OAC})_{2}$, and $9 \mathrm{~mL} \mathrm{OA}$ in a $100 \mathrm{ml}$ flask, which is heated to $150^{\circ} \mathrm{C}$ in flowing high-purity argon for 30min. Then, $20 \mathrm{ml}$ of ODE was added to the flask and the temperature was elevated to $305{ }^{\circ} \mathrm{C}$. At this temperature, $6 \mathrm{~mL}$ of Se precursor was swiftly injected into the reaction flack. The reaction temperature was kept at $305^{\circ} \mathrm{C}$ for $30 \mathrm{~min}$ for the core growth. Then the Se precursor II were injected dropwise into the reaction solution at a rate of $10 \mathrm{~mL} \mathrm{~h}^{-1}$ using a syringe pump at $305^{\circ} \mathrm{C}$. For the consecutive overcoating of the $\mathrm{ZnS}$ shell, a desired amounts of $\mathrm{Zn}(\mathrm{OA})_{2}$ and octanethiol (1.4 equivalent amount refers to $\left.\mathrm{Zn}(\mathrm{OA})_{2}\right)$ were added dropwise into the reaction solution at a rate of $5 \mathrm{~mL} \mathrm{~h}^{-1}$ using a syringe pump. Once the reaction was completed, the temperature was brought down to room temperature. QDs were purified six times by following a centrifugation precipitation/dispersion method using a mixed solvent of hexane/ ethanol (1/1 in volume ratio). Finally, the purified QDs were redispersed into hexane or chloroform for optical 
characterization, post-treatments, and the preparation of QD films.

Fabrication of QLEDs: The devices are composed of indium tin oxide (ITO, $100 \mathrm{~nm}$ ), PEDOT:PSS (35 nm), TFB (25 nm), core/shell QDs (18 nm), ZnO nanoparticles (NPs, $55 \mathrm{~nm}$ ), and aluminum (Al, $100 \mathrm{~nm}$ ) layers. ZnO NPs were synthesized by a solution-precipitation process using $\mathrm{Zn}$ acetate and $\mathrm{TMAH}^{32}$. For a typical synthesis, a solution of zinc acetate in DMSO $(0.5 \mathrm{~m})$ and $30 \mathrm{~mL}$ of TMAH in ethanol $(0.55 \mathrm{~m})$ were mixed and stirred for $1 \mathrm{~h}$ in ambient air. Then $\mathrm{ZnO}$ NPs were washed and dispersed in ethanol. QLEDs were fabricated on glass substrates coated with ITO with a sheet resistance of $\approx 15 \Omega \mathrm{sq}^{-1}$. The substrates were cleaned with deionized water, acetone, and isopropanol, consecutively for $15 \mathrm{~min}$ each. These substrates were spin-coated with PEDOT:PSS (AI 4083) and baked at $150{ }^{\circ} \mathrm{C}$ for $15 \mathrm{~min}$ in air. The coated substrates were transferred to a $\mathrm{N}_{2-}$ filled glove box for spin-coating of TFB, QDs, and ZnO NPs. As hole transport layer, TFB were spin-coated using $1.5 \mathrm{wt} \%$ in chlorobenzene ( $2000 \mathrm{rpm}$ for $30 \mathrm{~s}$ ), followed by baking at $110{ }^{\circ} \mathrm{C}$ for $30 \mathrm{~min}$. Then, it was spin-coated by QDs (15 mg mL-1, in toluene) and $\mathrm{ZnO}$ NPs (30 mg mL $\mathrm{mL}^{-1}$, in ethanol), followed by baking at $80{ }^{\circ} \mathrm{C}$ for $30 \mathrm{~min}$. The spin speed was $2500 \mathrm{rpm}$ for the QD layer and $3000 \mathrm{rpm}$ for the $\mathrm{ZnO}$ layer to achieve layer thickness of $\approx 18$ and $\approx 30 \mathrm{~nm}$, respectively. These multilayer structure samples were then loaded into a custom high-vacuum deposition chamber (background pressure, $\approx 3 \times 10^{-7}$ torr) to deposit the top $\mathrm{Al}$ cathode (100 nm thick) patterned by an in situ shadow mask to get an active device area of $4 \mathrm{~mm}^{2}$.

Characterization and instrumentation: UV-visible absorption and PL spectra were measured by an Ocean Optics spectrophotometer (model PC2000-ISA). All PLQY data of QDs were collected by using an Ocean Optics USB2000 spectrometer and an Ocean Optics ISP-50-8-I integrating sphere. Time-resolved fluorescence spectra were measured via the time-correlated single-photon counting method using a JY HORIBA FluoroLog-3 fluorescence spectrometer with a $405 \mathrm{~nm}$ picosecond pulsed diode laser with $1 \mathrm{MHz}$ repetition rate and $\sim 200 \mathrm{ps}$ pulse duration. The optical density (OD) values of the QD samples at the excitation wavelength were all adjusted to be in the range of 0.02-0.05. Transmission electron microscopy (TEM) studies were performed using a JEOL JEM-2010 electron microscope operating at $200 \mathrm{kV}$. Aberration-corrected high-angle annular dark-field scanning transmission electron microscopy (HAADF-STEM) images were obtained on a JEOL ARM200F operated at $200 \mathrm{kV}$. The energy dispersive spectroscopy (EDS) mappings were carried out by four symmetrically designed EDS detectors equipped on FEI Talos 
F200X. The cross-section images of the QLEDs were collected using FEI Talos F200X TEM. The phase and the crystallographic structure of the QDs were investigated by X-ray diffraction (XRD, D8-ADVANCE). The J-V characteristics of the QLEDs were analysed using an Agilent $4155 \mathrm{C}$ semiconductor parameter analyser with a calibrated Newport silicon diode under ambient conditions.

5 The luminance was calibrated using a Photo Research spectroradiometer (PR735). The electroluminescence spectra were obtained with an Ocean Optics spectrometer (USB2000, relative irradiance mode) and a Keithley 2400 source meter Calculations of the EQE. The EQE is calculated according to the formula below by assuming that the emission obeys a Lambertian profile ${ }^{3}$ whose validity is established later:

$$
\eta_{E Q E}=\frac{\pi L e}{K_{m} h c J} \frac{\int I(\lambda) \lambda \mathrm{d} \lambda}{\int I(\lambda) V(\lambda) \mathrm{d} \lambda}
$$

where $e$ is the electron charge, $h$ is the Planck constant, $c$ is the velocity of light and $\mathrm{K}_{m}=683 \mathrm{~lm}$ $\mathrm{W}^{-1}$ is the maximum luminous efficacy. The current density $J$ is measured from the $J-V$ characteristics using an Agilent $4155 \mathrm{C}$ semiconductor parameter analyser with a calibrated Newport silicon diode under ambient conditions. $I(\lambda)$ is the relative electroluminescence intensity at wavelength $\lambda$, obtained from the electroluminescence spectrum measured with an Ocean Optics spectrometer (USB2000, relative irradiance mode) supplied by a Keithley 2400 source meter, and $\mathrm{V}(\lambda)$ is the normalized photonic spectral response function. $\mathrm{L}$ is the total luminance, which is calibrated using a PR735.

Monte Carlo simulations: The charge injection currents in QLEDs can be described by driftingdiffusion model ${ }^{19}$. We are particularly interested in the case when the bias voltage is around the turn-on voltage, because the built-in field is offset by the bias voltage, the drift current is negligible, only diffusion current is considered here. According to the Fick's first law of diffusion, the diffusion carrier current density can be written as:

$$
\begin{aligned}
& J_{e}=\frac{P_{E \rightarrow Q} n_{E T L}-P_{Q \rightarrow E} n_{Q D}}{S \cdot \Delta t} \\
& J_{h}=\frac{P_{H \rightarrow Q} p_{H T L}-P_{Q \rightarrow H} p_{Q D}}{S \cdot \Delta t}
\end{aligned}
$$

Where $P_{E \rightarrow Q}\left(P_{Q \rightarrow E}\right)$ is the probability for electrons to transport from ETL (QD layer) to QD (ETL), and $P_{H \rightarrow Q}\left(P_{H \rightarrow Q}\right)$ is the probability for holes to transport from HTL (QD layer) to QD (HTL). 
$n_{E T L / H T L / Q D}$ and $p_{E T L / H T L / Q D}$ are the counts of electrons and holes in the ETL/HTL/QD layers, respectively. In single charge conductance measurements (Extended Data Figure 2), with given bias, similar single carrier currents are observed in both two devices using monolayers of large and regular QD, therefore, we assume $P_{E \rightarrow Q}$ and $P_{H \rightarrow Q}$ are also similar in two types of devices. We

5 further assumed $P_{E \rightarrow Q}=P_{Q \rightarrow E}=P_{n}$, and $P_{H \rightarrow Q}=P_{Q \rightarrow H}=P_{p}$ to simplify our calculation. Thus, the numbers of injected electron $\left(\mathrm{N}_{e}\right)$ and hole $\left(N_{h}\right)$ can be described as:

$$
\begin{aligned}
& \mathrm{N}_{e}=J_{e} \cdot S \cdot \Delta t=P_{n} \Delta n=P_{n}\left(n_{E T L}-n_{Q D}\right), \\
& N_{h}=J_{h} \cdot S \cdot \Delta t=P_{p} \Delta p=P_{p}\left(p_{H T L}-p_{Q D}\right) .
\end{aligned}
$$

The irradiative recombination current in QLED is generated by direct vertical charge injection and lateral charge migration, since the mobility in QD is low, the former is the dominant contributor. We use $100 \times 100$ and $56 \times 56$ matrix to represent large $(18 \mathrm{~nm})$ and regular $(10 \mathrm{~nm})$ QDs in QLED with the same area, and set $n_{Q D}=p_{Q D}=0$, and $n_{E T L}=n_{H T L}=1000$ as the initial condition. $P_{n}$ and $P_{p}$ are both set to be 0.01 . We use injected carrier numbers divided by QD population in the matrix as injection probability:

$$
P_{l e}=\frac{\mathrm{N}_{l e}}{56 \times 56}, P_{l h}=\frac{N_{l h}}{56 \times 56}, P_{r e}=\frac{\mathrm{N}_{r e}}{100 \times 100}, \text { and } P_{r h}=\frac{\mathrm{N}_{r h}}{100 \times 100} .
$$

Where $\mathrm{N}_{l e}, N_{l h}, \mathrm{~N}_{r e}$, and $\mathrm{N}_{r h}$ denotes the number of electron and holes injected into regular and large QDs, respectively. The corresponding injection probabilities are denoted by $P_{l e}, P_{l h}, P_{r e}$ and $P_{r h}$, respectively.

We have further taken columbic interaction into consideration during the charge injection process, a $60 \mathrm{meV}$ exciton binding energy was adopted in our calculations, according to the Boltzmann distribution, this will cause around 10 times difference in terms of injection probability. When there is an electron in a QD, the probabilities of injecting another electron into large and regular QD are denoted as $P_{l e e}$ and $P_{\text {ree }}$, respectively, which are 10 times lower than those empty QDs. Conversely, the probabilities of injecting another hole are 10 times higher, which are denoted as $P_{l e h}$ and $P_{r e h}$, respectively. For the QDs that are positively charged, the probability of injecting one more hole into large $\left(P_{l h h}\right)$ and regular $\left(P_{r h h}\right)$ QDs are also 10 times smaller, and the probability of injecting another electron are 10 times higher (denoted as $P_{\text {lhe }}$ and $P_{r h e}$ for the large and regular 
QDs, respectively).

$$
\begin{aligned}
& P_{l e e}=\frac{P_{l e}}{10}, P_{l h h}=\frac{P_{l h}}{10}, \quad P_{l e h}=P_{l h e}=10 *\left[\min \left(P_{l e}, P_{l h}\right)\right], \\
& P_{r e e}=\frac{P_{r e}}{10}, P_{r h h}=\frac{P_{r h}}{10}, P_{r e h}=P_{r h e}=10 *\left[\min \left(P_{r e}, P_{r h}\right)\right] .
\end{aligned}
$$

Then we start Monte Carlo simulation. In each cycle, we generate a random probability and compare with the probabilities calculated above to determine whether a charge is injected or not. At the end of one cycle, we let charge to migrate among different QDs, during which some excitons might be generated. Then we'll count the electron and hole population and go back to calculate the number of injected charge. A full cycle is considered as a unit time, the carrier numbers and exciton generation rates are recorded after each cycle. It is noted that the electron and hole injection are assumed to be balanced in Figure 2, to investigate whether imbalanced injection will affect the relative exciton generation rate, we slowed down the hole injection by up to 10 times and found the relative exciton generation rate largely remains constant (Extended Data Figure 10).

Out-coupling efficiency calculation: A transfer-matrix-method ${ }^{34,35}$ is used to calculate the outcoupling efficiencies of QLED with two different sizes, and the parameters used in calculation are listed in the Extended data table 1. Extended Data Fig. 6 shows the calculated emitting power distributions versus the wave-vectors, the out-coupling efficiencies are indicated by their area proportions, which are $22.4 \%$ and $23.9 \%$ for regular - and large- QD devices, respectively.

\section{Data availability.}

The data that support the findings of this study are available from the corresponding author upon reasonable request.

31. Shen, H. et al. High quality synthesis of monodisperse zinc-blende CdSe and CdSe/ZnS nanocrystals with a phosphine-free method. CrystEngComm 11, 1733-1738 (2009).

33 Okamoto, S. et al. Simple measurement of quantum efficiency in organic electroluminescent devices. Jpn. J. Appl. Phys 40, 783-784 (2001). in modes of arbitrary planar structures. J. Opt. Soc. Am. A 15, 1192-1201(1998).

35 Cho, C. \& Greenham, N. C. Computational study of dipole radiation in re-absorbing perovskite semiconductors for optoelectronics. Adv. Sci. 8, 2003559 (2020). 


\section{Supplementary Files}

This is a list of supplementary files associated with this preprint. Click to download.

- R40Extendeddata.docx 